\title{
Isotope and fast ions turbulence suppression effects: consequences for high- $\beta$ ITER plasmas
}

\author{
J. Garcia ${ }^{1}$, T. Görler ${ }^{2}$, F.Jenko ${ }^{2}$ \\ ${ }^{1}$ CEA, IRFM, F-13108 Saint-Paul-lez-Durance, France \\ ${ }^{2}$ Max Planck Institute for Plasma Physics,Boltzmannstr. 2, 85748 Garching, Germany
}

E-mail contact of main author: jeronimo.garcia@cea.fr

\begin{abstract}
The impact of isotope effects and fast ions on microturbulence is analyzed by means of non-linear gyrokinetic simulations for an ITER hybrid scenario at high beta obtained from previous integrated modelling simulations with simplified assumptions. Simulations show that ITER might work very close to threshold and in these conditions significant turbulence suppression is found from DD to DT plasmas. Electromagnetic effects are shown to play an important role for the onset of this isotope effect. Additionally, even external ExB flow shear, which is expected to be low in ITER, has a stronger impact on DT than in DD. The fast ions generated by fusion reactions can additionally reduce turbulence even more although the impact in ITER seems weaker than in present-day tokamaks.
\end{abstract}

\section{Introduction}

Optimizing thermal energy confinement time is essential in order to maximize the generation of fusion energy in future tokamak devices as ITER for which the alpha power is going to be the main heating mechanism. For this purpose, controlling and eventually suppressing heat transport, which is one the main mechanisms leading to significant thermal energy losses, is an essential step towards this goal. Recently, the benefits of high beta on thermal energy confinement have been demonstrated both in dedicated JET power scans and by means of non-linear gyrokinetic simulations, which also showed a significant impact of the fast-ion population in this turbulence reduction. Additionally, an isotope effect has been identified in such conditions due to the concomitant interplay of zonal flows, electromagnetic effects and mass, which is enhanced by the different impact of ExB flow shear on the different isotope species [1]. Therefore, a natural question is what will be the impact of a combination of all these effects on a high performance ITER plasma with a significant contribution of alpha power and how sensitive are the results on the assumptions made for the usual ITER scenario considered in the field. For that purpose, linear and non-linear gyrokinetic analyses on Ion Temperature Gradient (ITG) dominant plasmas are indispensable in order to address the impact of turbulence suppression effects on the future tokamak reactor. Additionally, the results obtained can provide experimental guidelines to present-day experimental campaigns focusing on ITER relevant conditions, as the expected JET-DT campaign [2,3].

In order to accomplish this goal, in this paper an ITER scenario previously modelled in an integrated modelling framework with the CRONOS [4] and DINA-CH codes [5], is used as a basis. It corresponds to a so called hybrid regime with high input power and $\beta$, with $\beta=$ $2 \mu_{0}<P>/ B^{2}$, where $\mathrm{P}$ is the plasmas pressure and $\mathrm{B}$ the magnetic field. A summary of the main plasma parameters can be found in [5] together with the plasma equilibrium details. 


\section{Codes used and modelling set-up}

The gyrokinetic code GENE [6] has been used for computing linear and nonlinear microturbulence characteristics in the core plasma region. All simulations are local and they include kinetic electrons, collisions and electromagnetic effects. The code employs field aligned coordinates, where $\mathrm{x}$ is the radial coordinate, $\mathrm{z}$ is the coordinate parallel along the field, and y is the binormal coordinate. Grid parameters were as follows: perpendicular box sizes $[\mathrm{Lx}, \mathrm{Ly}]=[153,125]$ in units of the ion Larmor radii, perpendicular grid discretization $[\mathrm{nx}, \mathrm{nky}]=[192,32], 32$ point discretization in the parallel direction, 48 points in the parallel velocity direction, and 12 magnetic moments. The instability linear growth rate $\gamma$ and the external ExB flow shear rate, $\gamma_{E x B, \text { ext }}=r / q d \Omega / d r$, with $\Omega$ the toroidal angular velocity, are in units of $\mathrm{C}_{\mathrm{s}} / \mathrm{R}$, with $C_{s}=\sqrt{T_{e} / m_{D}}$ and $\mathrm{T}_{\mathrm{e}}$ the electron temperature, $\mathrm{m}_{\mathrm{D}}$ the deuterium mass and $\mathrm{R}$ the tokamak major radius. Both $\delta \mathrm{B}_{\perp}$ and $\delta \mathrm{B}_{\|}$fluctuations were considered. As representative of the inner core region, the dimensionless parameters of the discharge at $\rho=0.33$, with $\rho=\sqrt{\varphi}$ and $\varphi$ the normalized toroidal flux, fed into the gyrokinetic calculations are summarized in Table I. Although the fast ion densities associated with the fusion $\alpha$-particles and with the NBI beams are low, $\mathrm{n}_{\text {fast } \alpha} / \mathrm{n}_{\mathrm{e}} \sim 0.009$ and $\mathrm{n}_{\text {fast,beams }} / \mathrm{n}_{\mathrm{e}} \sim 0.006$, their pressure (and pressure gradient), is not negligible as their energy, obtained from the Monte Carlo code SPOT [7] slowing-down distribution function, is quite high, $\mathrm{T}_{\mathrm{f}, \alpha}=1.1 \mathrm{MeV}$ and $\mathrm{T}_{\mathrm{f}, \text { beams }}=0.55 \mathrm{MeV}$.

TABLE I: DISCHARGE DIMENSIONLESS PARAMETERS AT $\rho=0.33$ USED AS INPUT IN SIMULATIONS. S IS THE MAGNETIC SHEAR, Q THE SAFETY FACTOR, TE AND TI THE ELECTRON AND ION TEMPERATURES RESPECTIVELY, R/L $/ \mathrm{L}_{\mathrm{TI}}=-R d T_{i} / d \rho / T_{i}$,

$$
\mathrm{R} / \mathrm{L}_{\mathrm{TE}}=-R d T_{e} / d \rho / T_{e} \text { AND R/L } / \mathrm{LE}=-R d N_{e} / d \rho / N_{e}
$$

\begin{tabular}{c|cccccccccccc}
\multicolumn{2}{c}{$\mathrm{s}$} & $\mathrm{q}$ & $\mathrm{T}_{\mathrm{e}} / \mathrm{T}_{\mathrm{i}}$ & $\mathrm{R} / \mathrm{L}_{\mathrm{Ti}}$ & $\mathrm{R} / \mathrm{L}_{\mathrm{Te}}$ & $\mathrm{R} / \mathrm{L}_{\mathrm{Ne}}$ & $\mathrm{T}_{\mathrm{f}, \text { beams }} / \mathrm{T}_{\mathrm{e}}$ & $\mathrm{R} / \mathrm{L}_{\mathrm{Tf} \text { beams }}$ & $\mathrm{R} / \mathrm{L}_{\mathrm{Nf}, \text { beams }}$ & $\mathrm{T}_{\mathrm{f}, \alpha} / \mathrm{T}_{\mathrm{e}}$ & $\mathrm{R} / \mathrm{L}_{\mathrm{Tf}, \alpha}$ & $\mathrm{R} / \mathrm{L}_{\mathrm{Nf}, \alpha}$ \\
\hline ITER & 0.24 & 1.17 & 1.09 & 4.5 & 2.9 & 1.9 & 22.5 & 1.85 & 13.1 & 41.3 & 0.94 & 9.23
\end{tabular}

\section{Linear gyrokinetic analysis}
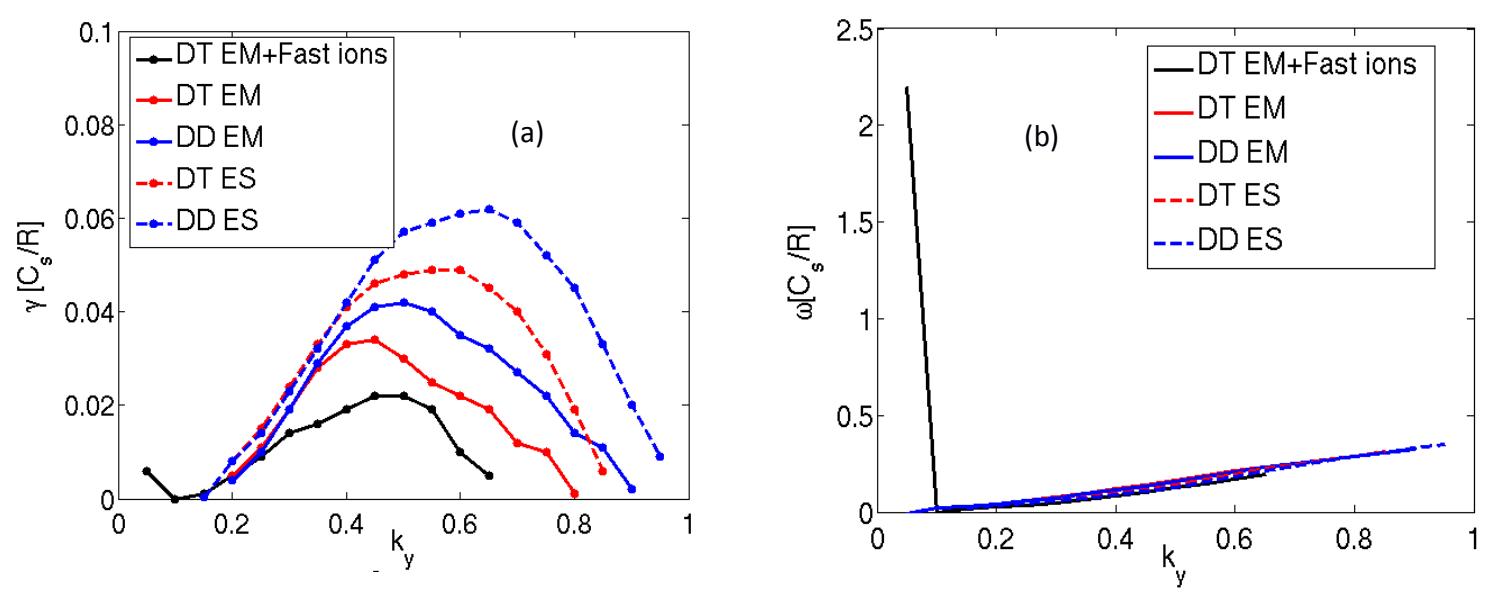

FIG. 1 Linear growth rates for DD and DT including electromagnetic effects (EM) or performing electrostatic (ES) simulations (left) Frequency for the previous cases (right). 

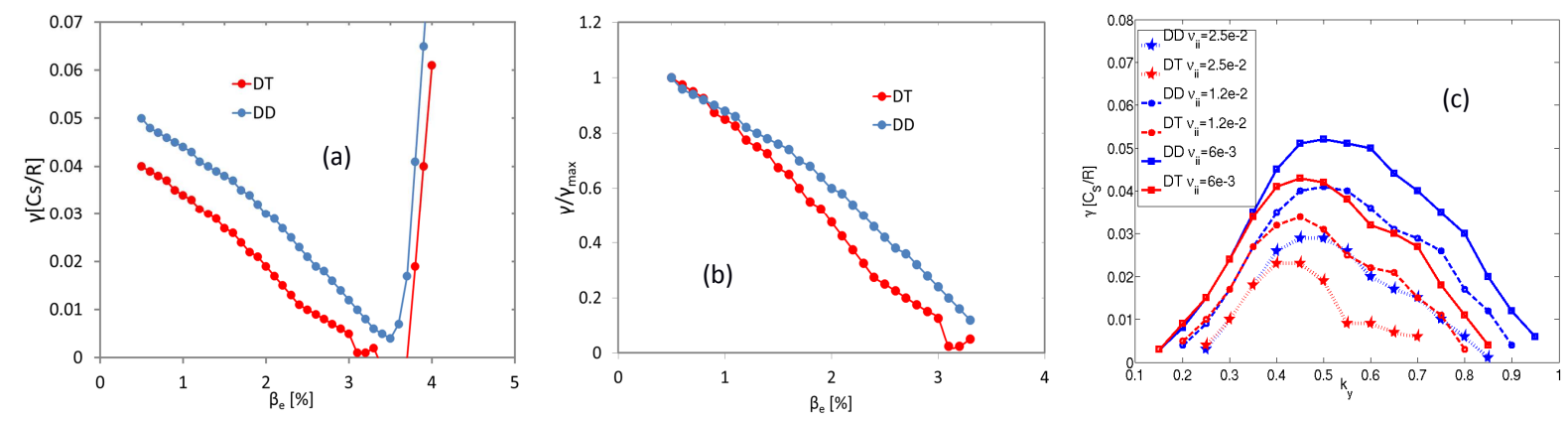

FIG. 2 Linear $\beta_{e}$ scan at $\mathrm{k}_{\mathrm{y}}=0.5$ for DD and $\mathrm{T}$ (a). $\gamma / \gamma_{\max }$ at $\mathrm{k}_{\mathrm{y}}=0.5$ dependence on $\beta_{e}$ for DD and DT (b) Linear collisionality scan for DD and DT (c)

At first, a linear analysis has been carried out for different plasma configurations without including the effect of ExB flow shear. In figure $1 \mathrm{a}$ and $1 \mathrm{~b}$, the linear growth rates and frequencies are shown. Over a wide normalized wavenumber range, $k_{y}=\hat{k}_{y} \rho_{s}$ where $\rho_{s}$ is the ion gyroradius normalized with respect to the sound speed, ITG modes are unstable except for the full DT standard case including the fast ion population for which a high frequency mode appears at $\mathrm{k}_{\mathrm{y}}=0.05$. This mode is identified here as Alfven Beta Eigenmode (BAE)/Kinetic Ballooning Modes (KBM) [8], mainly driven by the energetic particles, therefore, as it happens for JET hybrid scenarios with high confinement [9], it lies in the ITG- KBM/BAE boundary. When including the fast ion population there is a significant decrease of $30 \%$ of the maximum growth rate compared to the case without fast ions. This shows that, from the linear point of view, a similar reduction can be expected in ITER and JET. When removing the fast ion component, this mode disappears and only ITG modes are obtained.

From the simulations without the energetic ion population, it is clear that the maximum growth rate, $\gamma_{\max }$, is lower for DT, $\gamma_{\max , D T}=0.034\left[C_{S} / R\right]$, than for $\mathrm{DD}, \gamma_{\max , D D}=$ $0.042\left[C_{S} / R\right]$, however this trend closely follows the Gyro-Bohm scaling as $\gamma_{\max , D T} \sim \gamma_{\max , D D} \sqrt{m_{D D} / m_{D T}}$ something expected from the fact that the ITG growth rate scales with the thermal ion velocity $v_{t h, i}=\sqrt{T_{i} / m_{i}}$. Following the scaling of the gyroradius with mass there is also a shift towards lower values of the $\mathrm{k}_{\mathrm{y}, \max }$ corresponding to $\gamma_{\max }$ for DT. When comparing the growth rates with (EM) and without (ES) electromagnetic effects the ratio $\gamma_{\max , D D} / \gamma_{\max , D T}$ remains constant, showing that no significant electromagnetic effects are found linearly for the maximum growth rate.

The impact of electromagnetic effects and the ion-ion collisionality, $v_{i i}$, has been analyzed by performing a scan on $\beta_{e}=2 \mu_{0}<P_{e}>/ B^{2}$ at $\mathrm{k}_{\mathrm{y}}=0.5$, with $P_{e}$ the electron pressure, and by perfroming a linear scan at three different $v_{i i}$ and at nominal $\beta_{e}$ as shown in figure 2.

Regarding electromagnetic effects, the ratio $\gamma_{D D} / \gamma_{D T}$ increases with $\beta_{e}$ from $\gamma_{D D} / \gamma_{D T}=$ 1.25 for $\beta_{e}=0.5 \%$ up to $\gamma_{D D} / \gamma_{D T}=2.4$ at $\beta_{e}=3.0 \%$. From that point, when approaching the ITG-KBM transition, $\gamma_{D T}$ is significantly lower than $\gamma_{D D}$ and the transition to KBM occurs at lower $\beta_{e}$. The trend is clearly observed when $\gamma / \gamma_{\max }$ is plotted for both DD and DT against $\beta_{e}$. For the DT mixture, $\gamma / \gamma_{\max }$ decreases much faster with $\beta_{e}$ than for DD. These findings suggest a different electromagnetic behavior of the DT and DD mixtures which can lead to different non-linear behavior as well $[9,10]$. This possibility will be checked in the next section.

Regarding the collisionality scan, $\gamma_{D D}$ and $\gamma_{D T}$ both increase with decreasing ion collisionality $v_{i i}$. However, we do not see any particular impact of the ion mass on the maximum growth rate and the relation $\gamma_{\max , D T} \sim \gamma_{\max , D D} \sqrt{m_{D D} / m_{D T}}$ also holds for this scan. Interestingly, the 
fact that the growth rates are significantly changed by collisionality might indicate that subdominant trapped modes may also play a role on these simulations. This point will be clarified in the following sections.

\section{Non-linear gyrokinetic analysis of heat flux}

Non-linear simulations have been also performed for the three reference cases, DT including the fast ion population (1), DT (2) and DD (3) and several non-linear scans have been performed with the aim of analyzing the sensitivity of the results to the different assumptions. The effect of the external ExB flow shear, $\gamma_{\mathrm{ExB} \text {,ext }}$, which is known to quench turbulence in the ITG domain and the consequent parallel velocity gradient (PVG) which can destabilize it has been also induced in these simulations. Due to the uncertainties on the external torque on ITER, which is the main source of $\gamma_{\mathrm{ExB}, \mathrm{ext}}$, there is no consensus on the value for this parameter, however it is expected to be considerably lower than in present day tokamaks due to the low torque applied. Therefore, a reduced value, in agreement with ITER integrated modelling simulations [11], has been assumed, $\gamma_{\mathrm{ExB}, \mathrm{ext}}=0.01$. A particular scan on this parameter has been carried out as well.

\begin{tabular}{c|cc|}
$\#$ & Case & $\mathrm{Q}_{\mathrm{i}}\left(\mathrm{kW} / \mathrm{m}^{2}\right)$ \\
\hline $\boldsymbol{1}$ & DT+fast ions & 84 \\
$\boldsymbol{2}$ & $D T$ & 154 \\
$\mathbf{3}$ & $D D$ & 271 \\
$\mathbf{4}$ & DT lumping & 176 \\
\cline { 3 - 3 } & &
\end{tabular}

\section{TABLE II: NON-LINEAR HEAT FLUXES OBTAINED INCLUDING ELECTROMAGENTIC EFFECTS AND EXTERNAL EXB.}

A summary of the heat fluxes is shown in table II. The lowest heat flux is indeed obtained for the case including the fast ion component, $Q_{i, D T, f a s t}=86 \mathrm{~kW} / \mathrm{m}^{2}$ which is $50 \%$ lower than the one obtained without fast ions but still taking into account a DT mixture, $Q_{i, D T}=$ $154 \mathrm{~kW} / \mathrm{m}^{2}$, which means that the impact of the fast ion component is stronger non-linearly, as it happens in JET $[9,12]$. Finally, the highest heat flux is obtained for the DD mixture, $Q_{i, D D}=271 \mathrm{~kW} / \mathrm{m}^{2}$, clearly showing a departure from the expected Gyro-Bohm scaling. The strong impact of the fast ion component on these simulations could be weaker if nonMaxwellian rather than equivalent Maxwellian distribution functions are considered [13].

Interestingly, this ITER case has been found to be close to threshold. A non-linear $\mathrm{R} / \mathrm{L}_{\mathrm{Ti}}$ scan has been performed with the aim of analyzing the threshold and stiffness levels for the different mixtures and whether the result obtained are obtained just because of the closeness to threshold. The results, shown in Fig. 3, demonstrate that although the isotope effect found in this paper is stronger close to threshold, it is still present at higher $\mathrm{R} / \mathrm{L}_{\mathrm{Ti}}$. Actually, there is an up-shift of stiffness from DD to DT whereas the stiffness level remains similar. This upshift is similar to the one obtained in dedicated isotope experiments performed in the past in JT-60U [14]. On the other hand, when including the fast ions, there is an additional change of stiffness. In general, the amount of reduction of heat transport by the combined electromagnetic and fast ions effects is significant but lower than the one obtained for JET 
hybrid scenarios. As shown in [15], the fact that for ITER $T_{e}>T_{i}$ can be a reason for this weaker impact.
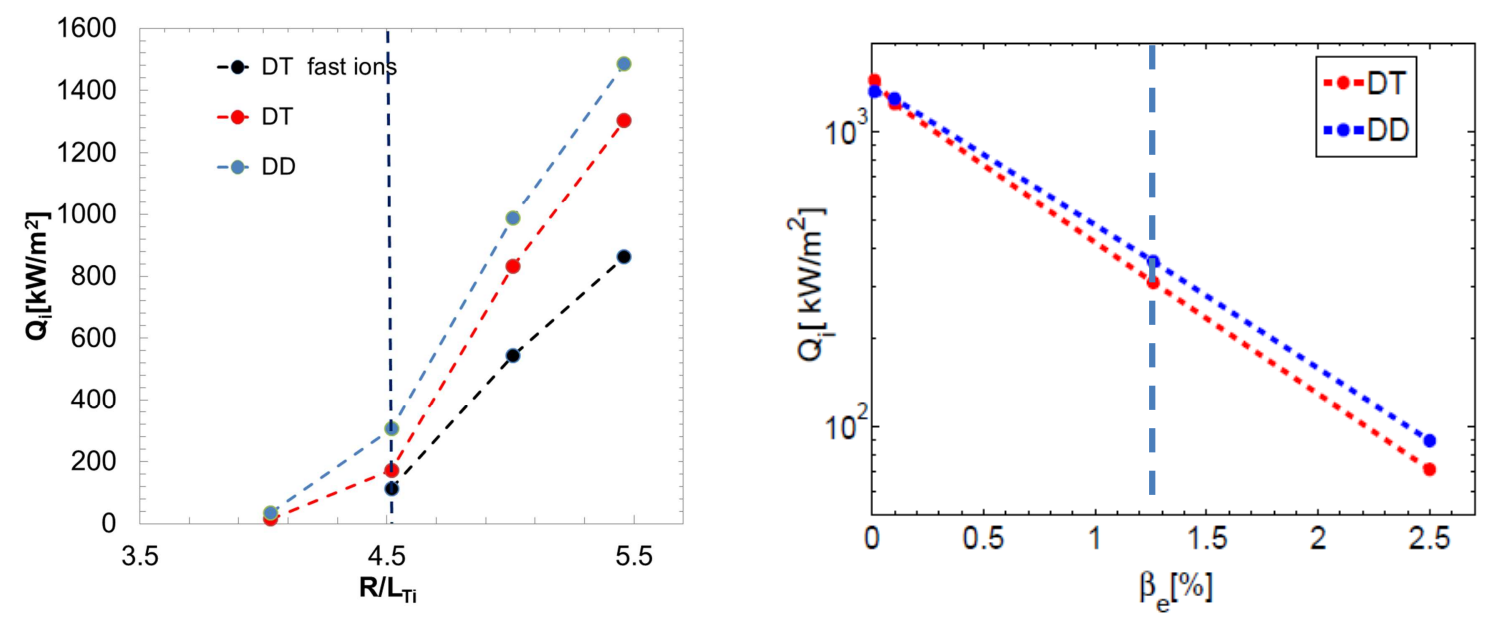

FIG. 3 Scan of the $Q_{i}$ dependence on $\mathrm{R} / \mathrm{L}_{\mathrm{Ti}}$ including ExB and electromagnetic effects (left) $Q_{i}$ scan on $\beta_{\mathrm{e}}$ for DD and DT excluding ExB effects (right) Dashed lines correspond to nominal values.

In order to investigate the impact of the different parameters used in the ITER hybrid scenario on the results obtained, in particular in DT vs DD mixtures, several non-linear scans have been performed on some parameters either known to play a role on the isotope effect, as ExB flow shear or electromagnetic effects, or some other which could play a role, as collisonality. Additionally, the role of multi ion species has been analyzed by performing a non-linear simulation lumping the DT mixture into a single ion species with effective mass 2.5 times the hydrogen mass, $\mathrm{m}_{\mathrm{eff}}=2.5 \mathrm{~m}_{\mathrm{H}}$.

As shown in table 2, the lumping of species has an effect on the heat fluxes. Whereas an isotope effect is still evident, as the heat flux obtained with the lumped species is $Q_{i, \text { lump }}=$ $176 \mathrm{~kW} / \mathrm{m}^{2}$ which is therefore lower than in DD, $Q_{i, D D}=271 \mathrm{~kW} / \mathrm{m}^{2}$ however it is higher than in DT $Q_{i, D T}=154 \mathrm{~kW} / \mathrm{m}^{2}$. Therefore, although lumping is not a bad approximation in these plasma conditions, the isotope effect seems to depend on the specific mass content and thus it is expected some difference in single vs multi on species plasmas.

A scan on $\beta_{e}$ (excluding external ExB shearing effects) is performed and shown in figure 3 (b). There is a stronger impact of electromagnetic effects for the DT mixture, which is strongly non-linear on $\beta_{e}$, whereas for $\beta_{e}<0.1 \%, Q_{i, D D} \geq Q_{i, D T}$, at $\beta_{e}=2.5 \%, Q_{i, D T}$ is nearly $30 \%$ lower than $Q_{i, D D}$. The interplay between electromagnetic effects, zonal flows and mass has been raised to explain such trend [1].

The scan on $\gamma_{\mathrm{ExB} \text {,ext, }}$ in figure 4 (in pure electrostatic conditions and including self-consistent PVG), shows as well significant differences between DD and DT species. Whereas at $\gamma_{\mathrm{ExB}, \mathrm{ext}}=0, Q_{i, D T}>Q_{i, D D}$ is obtained with $Q_{i, D T} / Q_{i, D D}$ following GyroBohm scaling, the trend is reversed with just $\gamma_{\mathrm{ExB}, \mathrm{ext}}=0.003$ which is ten times lower than $\gamma_{\max , D T}$. Indeed with $\gamma_{\mathrm{ExB}, \mathrm{ext}}=0.01$ the difference between DT and DD is further amplified. This scan shows that in ITG conditions the inclusion of just some external rotation can lead to a significant isotope effect as this point is particularly important for future isotope experiments, as the torque by the Neutral Beam Injection (NBI) heating system, which is usually used to heat the plasma, depends on the mass and therefore controlling such parameters is essential for having a control of $\gamma_{\text {ExB,ext }}$ in dedicated comparisons [3]. 
The impact of collisionality has been studied in the electromagnetic case and including $\gamma_{\text {ExB,ext}}=0.01$. As shown in figure 4 , following what was obtained already in the linear analysis, the heat fluxes increase with decreasing collisionality, however this behavior does not translate in a clear isotope effect as the DD flux is always higher than DT and the ration $Q_{i, D T} / Q_{i, D D}$ does not significantly change.
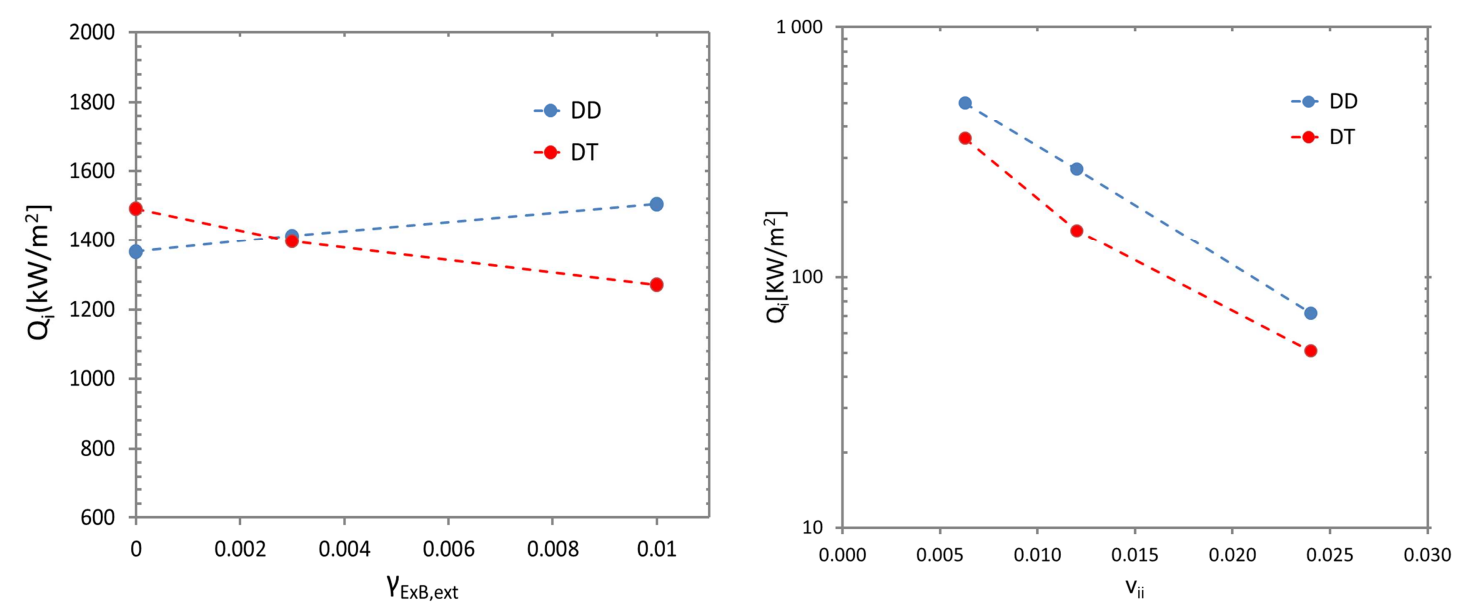

FIG. $4 Q_{i}$ scan on $\gamma_{E x B, \text { ext }}$ for DD and DT excluding electromagnetic effects (left) $Q_{i}$ scan on $v_{i i}$ for DD and DT including both electromagnetic and $\gamma_{E x B, e x t}$ effects (right)

\section{Role of trapped particles}
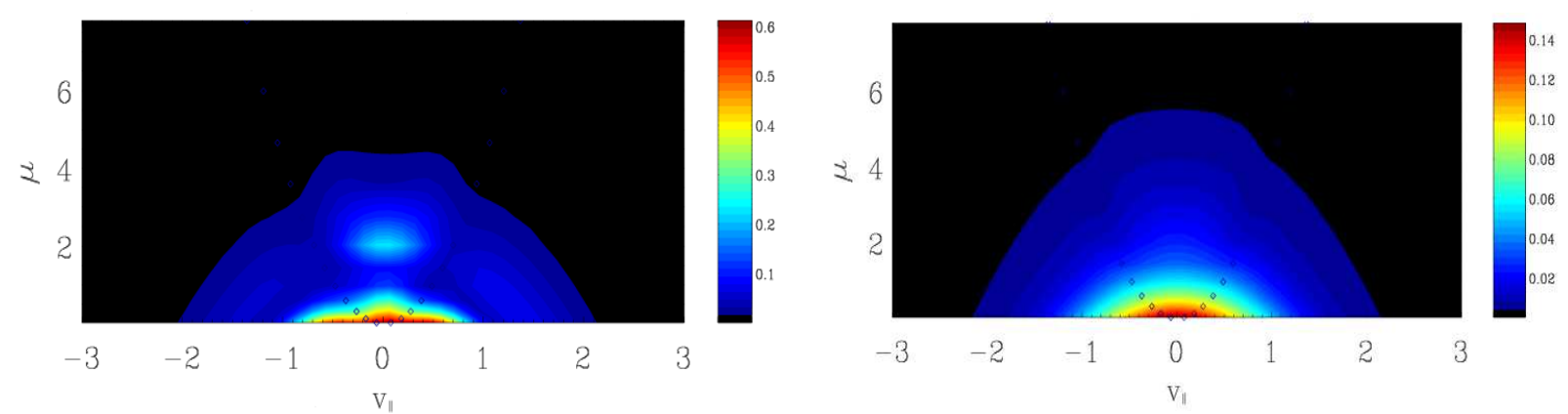

FIG. 5 Dependence of the distribution function on the velocity space (magnetic moment $\mu$ and the parallel velocity $v_{\|}$) for deuterium (left) and electrons (right). The trapped region is highlighted by using dots.

The strong sensitivity of the fluxes to collisions can be surprising taking into account that the most unstable micro-instabilities, as shown in section 2, are ITG modes which are known to be insensitive to collisions, though collisions can damp the zonal flows which in turn can reduce turbulence even in ITG dominated plasmas. In order to further understand whether these modes are playing a role, in figure 5 the dependence of the distribution function on the velocity space, i.e. the magnetic moment $\mu$ and the parallel velocity $v_{\|}$, is shown for deuterium and electrons.

Whereas for the electrons there is no significant evidence of trapped particles, suggesting that Trapped Electron Modes (TEM) are not playing a role in this plasma, the deuterium case (as it 
happens for tritium not shown here) there is a non-negligible sign of trapped ions. This may suggest a sub-dominant contribution of Trapped Ion Modes (TIM). This possibility links the results obtained in this paper with other studies in which the role of TEM have been highlighted to create an isotope effect through zonal flows [16, 17, 18, 19]. However, the exact role of these subdominant modes on the isotope effect found in this paper and their interaction with the mechanisms found to play a role is out of the scope of this paper and will be studied in the future.

\section{Flux spectra and fluctuations}

In order to understand the impact that the change of species has on the heat transport a dedicated analysis of the heat flux spectra has been carried out. The change from DD to DT is analyzed in figure 6 for the cases where electromagnetic effects and ExB flow shear are included, figure 6(a), and in the case of pure electrostatic simulation with no other effect figure 6 (b). In both cases there is a clear shift of the high $\mathrm{k}_{\mathrm{y}}$ and in particular of $\mathrm{k}_{\mathrm{y}, \max }$ towards lower values as the total mass content is increased, something that was already observed in the linear analysis. In the case of the electrostatic simulation, the shift is accompanied by an increasing of the amplitude intensity, which can explain the increasing heat flux obtained in DT with respect to DD. On the other hand, when both electromagnetic effects and ExB flow shear are included, in addition to the shift there is a significant reduction of the amplitude intensity of $\mathrm{k}_{\mathrm{y} \text {,max }}$. Interestingly, the shift towards lower $\mathrm{k}_{\mathrm{y}}$ is stronger for the case with electromagnetic effects and external ExB, however, the fluxes are lower.

The quantity $\sqrt{<|n|^{2}>/\left(n_{0} \rho^{*}\right)^{2}}$, with $n$ the fluctuating density and $n_{0}$ the equilibrium density, which represents the density fluctuations, is shown in figure 7 and the pattern is similar to the heat fluxes. In purely electrostatic conditions and no external ExB, the D and T fluctuations are higher than D in the DD plasmas. However, this trend is reversed when considering electromagnetic effects and external ExB.

The different patterns in the spectrum of the heat flux, the fluctuations, the correlation length or zonal flows [1] when changing the ion mass may indicate a new way of characterizing the isotope effect by means of detailed measurements of turbulence characteristics, rather than comparison of diffusivities which rely on the calculation of power balance fluxes.
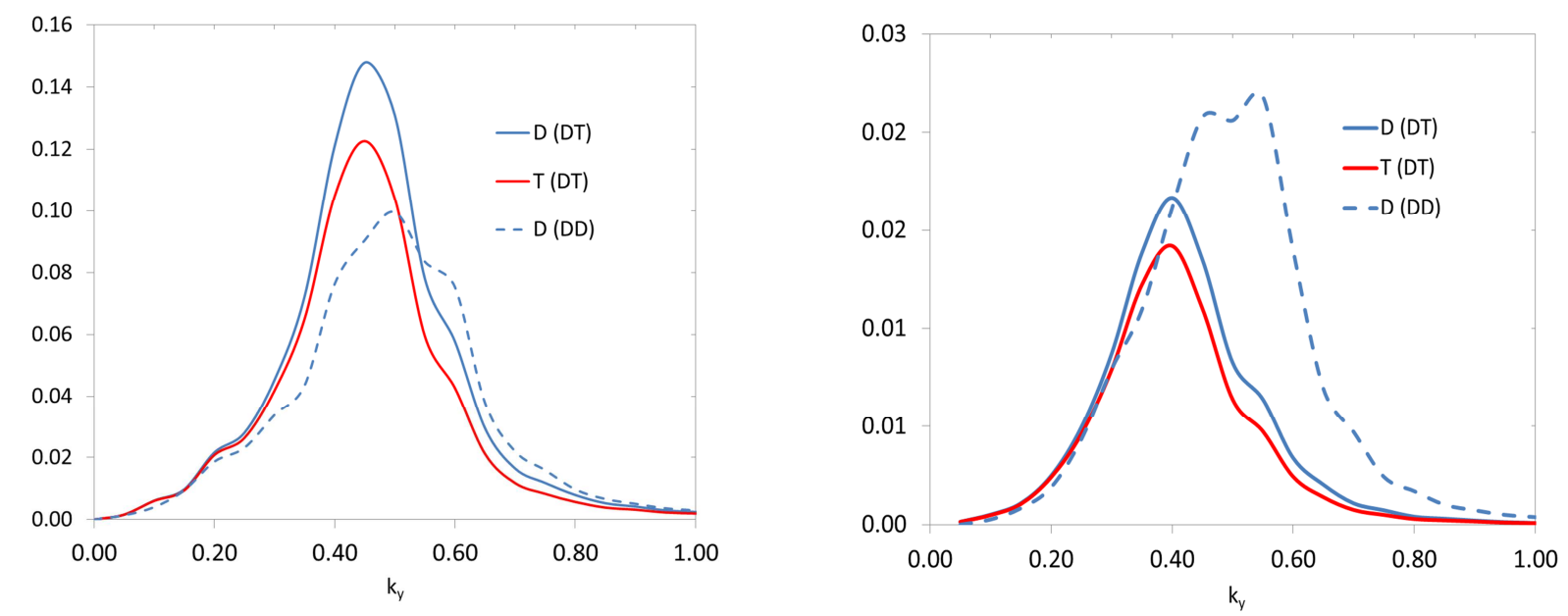

FIG. 6 Ion heat flux spectrum for the DD and DT simulations in electrostatic conditions and no external ExB (left). Ion heat flux spectrum for the DD and DT simulations in electromagnetic conditions and external ExB (right). 

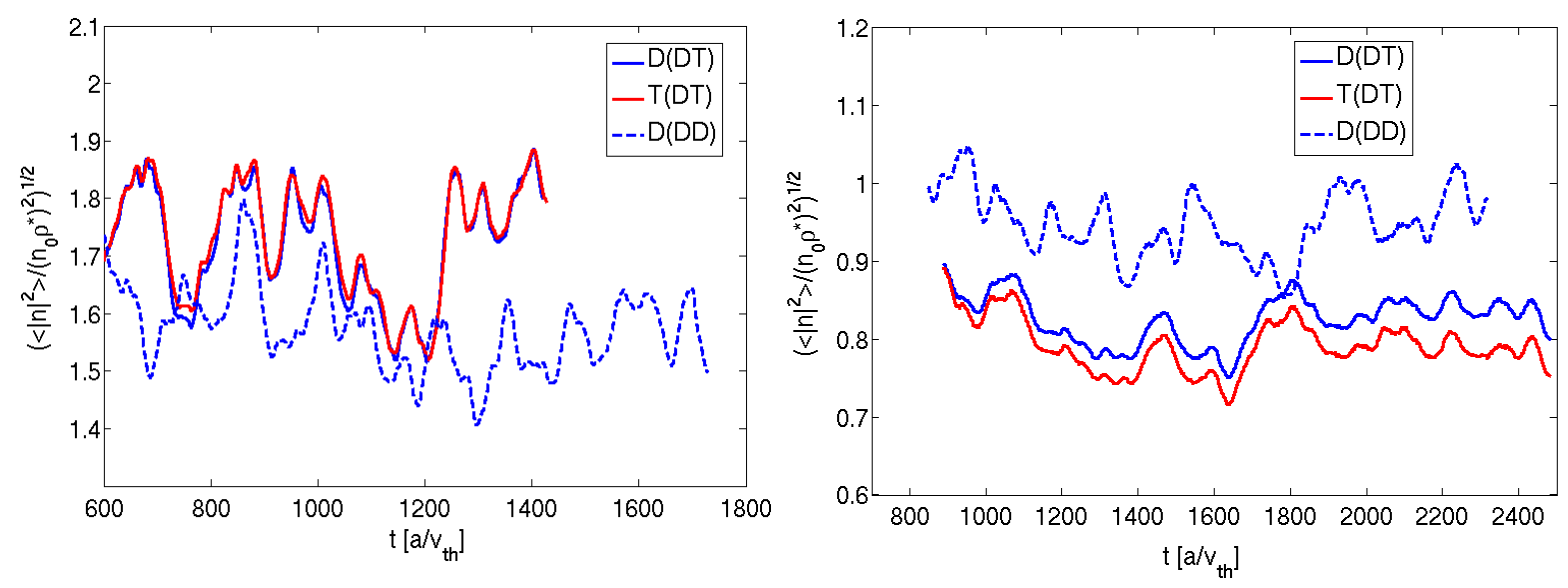

FIG. $7 \sqrt{<|n|^{2}>/\left(n_{0} \rho^{*}\right)^{2}}$ for the DD and DT simulations in electrostatic conditions and no external ExB (left) and for the DD and DT simulations in electromagnetic conditions and external ExB (right).

\section{The isotope effect in particle transport}

TABLE III: PARTICLE FLUX OBTAINED FOR THE CASES CONSIDERED IN THIS PAPER.

\begin{tabular}{c|cccc|}
$\#$ & Case & $\Gamma_{\mathrm{D}}\left(\mathrm{W} / \mathrm{eVm}^{-2}\right)$ & $\Gamma_{\mathrm{T}}\left(\mathrm{W} / \mathrm{eVm}^{-2}\right)$ & $\Gamma_{\text {Total }}\left(\mathrm{W} / \mathrm{eVm}^{-2}\right)$ \\
\hline $\mathbf{1}$ & $D T$ & 0.34 & 0.25 & 0.59 \\
$\mathbf{2}$ & $D D$ & 0.92 & & 0.92 \\
$\mathbf{3}$ & DT no effect & 2.36 & 1.07 & 3.43 \\
$\mathbf{4}$ & DD no effect & 2.6 & & 2.60 \\
\cline { 2 - 5 }
\end{tabular}

The impact of the isotope effect on particle transport has been also analyzed. In table III particle fluxes for $\mathrm{D}$ and $\mathrm{T}$ in $\mathrm{DD}$ and $\mathrm{DT}$ mixtures are compared for the cases in pure electrostatic conditions and no external ExB and full cases. An isotope effect has been also obtained for the particle transport, with a particle flux higher for DD, $\Gamma_{\mathrm{DD}}=0.92 \mathrm{~W} / \mathrm{eVm}^{-2}$, than

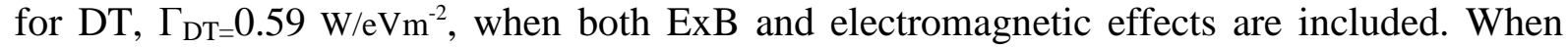
these effects are excluded then $\Gamma_{\mathrm{DT}}=3.43 \mathrm{~W} / \mathrm{eVm}^{-2}$ and $\Gamma_{\mathrm{DD}}=2.60 \mathrm{~W} / \mathrm{eVm}^{-2}$. Additionally, it is found that $\Gamma_{\mathrm{T}}$ is lower than $\Gamma_{\mathrm{D}}$ regardless the simulation condition. This is in agreement with previous isotope analyses [20]. All these facts suggest that density could have higher peaking in DT mixtures than in DD due to a better confinement and stronger impact of ExB [21-23]. However, the final density profile for both mixtures, and in particular the peaking, would depend as well on external factors not taken into account here, as the NBI fueling or the external torque which usually are quite different for $\mathrm{D}$ and $\mathrm{T}$ [3]. Therefore, the final results should be obtained by integrating all these ingredients in integrated modeling simulations. 


\section{Summary and conclusions}

The impact of a fast ion population and isotope effects has been analyzed for an ITER hybrid scenario by means of the gyrokinetic theory applying the GENE code. Whereas if they are isolated, electromagnetic effects, external ExB flow shear and fast ions effects are weak, the concomitant of such physical mechanisms in the DT hybrid regime can lead to a significant reduction of ITG turbulence compared to DD plasmas. This is of special importance as it is found that ITER hybrid will be close to threshold, something uncommon with respect to present-day plasmas.

The non-linear scans performed in this paper show the robustness of the results obtained, as with relatively low beta end external ExB a clear isotope effect appears. Something that, on the other hand, does not prevent the onset of a stronger effect in ITER plasmas with higher beta than the one assumed here.

Unlike most of the studies performed until now, which highlight the role of TEM, this effect is found here in ITG dominated plasmas. However, Trapped Ion Modes may play a role as found by analyzing the distribution function in the phase space velocity.

This feature, which is not common in present-day tokamak devices, together with the fact that the scenario analyzed is really close to threshold, as also found with the Gyro code [24] for the ITER baseline, aware against simplistic extrapolations to ITER from experimental results obtained in different regimes or from models which are not able to capture the complex physics behind such effects, as zonal-flows interplay with electromagnetic effects and mass and external ExB and PVG. Extra benchmarks of those models with non-linear gyrokinetic simulations in conditions close to ITER are recommended.

Finally, additional mechanisms have been found to modulate the isotope effect found here, as collisionality or the number of ion species. Together with other possible mechanisms found elsewhere, as the impurities [25], this shows that the complexity of this effect is significant. Therefore, additional studies both from theory and experimental sides, in a coordinated way, are required to finally shed light on this long-standing issue.

Acknowledgements. The simulations presented in this work were carried out using the HELIOS supercomputer system at Computational Simulation Centre of International Fusion Energy Research Centre (IFERC-CSC), Aomori, Japan, the MARCONI supercomputer at the Italian SuperComputing Resource Allocation (ISCRA) and the MARENOSTRUM supercomputer in the Barcelona Supercomputer Center (BSC) in the framework of the PRACE project ZONALGENE

\section{References}

[1] J. Garcia, T. Görler, F. Jenko and G. Giruzzi Nucl. Fusion 57014007 (2017)

[2] J. Paméla, F.Romanelli, M.L.Watkins, A.Lioure, G.Matthews, V.Philipps, T.Jones, A.Murari, A.Géraud, F.Crisanti, R.Kamendje Fusion Engineering Design 87, 590 (2007).

[3] J. Garcia, C. Challis, D. Gallart, L. Garzotti, T. Görler, D. King, M. Mantsinen and JET contributors, Plasma Phys. Control. Fusion 59, 014023 (2017).

[4] J.F. Artaud, V. Basiuk, F. Imbeaux, M. Schneider, J. Garcia, G. Giruzzi, P. Huynh, T. Aniel, F. Albajar, J.M. Ané, A. Bécoulet, C. Bourdelle, A. Casati, L. Colas, J. Decker, R. Dumont, L.G. Eriksson, X. Garbet, R. Guirlet, P. Hertout, G.T. Hoang, W. Houlberg, G. 
Huysmans, E. Joffrin, S.H. Kim, F. Köchl, J. Lister, X. Litaudon, P. Maget, R. Masset, B. Pégourié, Y. Peysson, P. Thomas, E. Tsitrone and F. Turco Nucl. Fusion 50, 043001 (2010).

[5] K. Besseghir, J. Garcia, J.F. Artaud, F. Imbeaux, R. R. Khayrutdinov, J. B. Lister, V. E. Lukash, P. Maget Plasma Phys. Control. Fusion 55, 125012 (2013).

[6] F. Jenko, W. Dorland, M. Kotschenreuther and B.N. Rogers, Phys. Plasmas 7, 1904 (2000).

[7] M. Schneider, L.-G. Eriksson, I. Jenkins, J.F. Artaud, V. Basiuk, F. Imbeaux, T. Oikawa, JET-EFDA contributors and ITM-TF contributors Nucl. Fusion 51, 063019 (2011).

[8] F. Zonca, L. Chen and R. A. Santoro Plasma Phys. Control. Fusion 38, 2011 (1996).

[9] J. Garcia, C. Challis, J. Citrin, H. Doerk, G. Giruzzi, T. Görler, F. Jenko, P. Maget and JET Contributors Nucl. Fusion 55, 053007 (2015).

[10] J. Citrin, J. Garcia, T. Görler, F. Jenko, P. Mantica, D. Told, C. Bourdelle, D. R. Hatch, G. M. D. Hogeweij, T. Johnson Plasma Phys. Control. Fusion 57014032 (2015).

[11] R.V. Budny, R. Andre, G. Bateman, F. Halpern, C.E. Kessel, A. Kritz and D. McCune Nucl. Fusion 48075005 (2008).

[12] J. Citrin, F. Jenko, P. Mantica, D. Told, C. Bourdelle, J. Garcia, J. W. Haverkort, G. M. D. Hogeweij, T. Johnson, and M. J. Pueschel Phys. Rev. Lett. 111, 155001 (2013).

[13] A. Di Siena, T. Görler, H. Doerk, J. Citrin, T. Johnson, M. Schneider, E. Poli and JET Contributors., Journal of Physics: Conference Series 775 (2016) 012003

[14] H. Urano, T. Takizuka, N. Aiba, M. Kikuchi, T. Nakano, T. Fujita, N. Oyama, Y. Kamada, N. Hayashi and the JT-60 Team Nucl. Fusion 53 (2013) 083003

[15] H Doerk, C Challis, J Citrin, J Garcia, T Görler, F Jenko and JET Contributors Plasma Phys Control Fusion 58 (2016) 115005

[16] T.S. Hahm, Lu Wang, W.X. Wang, E.S. Yoon, F.X. Duthoit Nucl. Fusion 53, 072002 (2013).

[17] A. Bustos, A. Bañón Navarro, T. Gorler, F. Jenko and C. Hidalgo Phys. Plasmas 22, 012305 (2015).

[18] M. Nakata, M. Nunami, H. Sugama, and T. H. Watanabe Phys. Rev. Lett. 118, 165002 (2017)

[19] P. Niskala, A. D. Gurchenko, E. Z. Gusakov, A. B. Altukhov, L. A. Esipov, M. Yu Kantor, T. P. Kiviniemi, D. Kouprienko, T. Korpilo, S. I. Lashkul, S. Leerink, A. A. Perevalov and R. Rochford Plasma Phys. Control. Fusion 59 (2017) 044010

[20] C. Estrada-Mila, J. Candy, and R. E. Waltz Phys. Plasmas 12, 022305 (2005).

[21] W. Buangam, J. Garcia, T. Onjun submitted to ppcf.

[22] J. Garcia, N. Hayashi, B. Baiocchi, G. Giruzzi, M. Honda, S. Ide, P. Maget, E. Narita, M.

Schneider, H. Urano, the JT-60U Team, the JET EFDA Contributors and the EU-ITM ITER Scenario Modelling Group Nucl. Fusion 54 (2014) 093010

[23] X Wang, S Mordijck, L Zeng, L Schmitz, T L Rhodes, E J Doyle, R Groebner, O Meneghini, G M Staebler and S P Smith Plasma Phys Control Fusion 58045026 (2016) 
[24] J. Candy and G.M. Staebler "Crucial role of zonal flows and electromagnetic effects in ITER turbulence simulations near threshold “ TH/P2-7 26th IAEA Fusion Energy Conference, Kyoto, Japan 17-22 October 2016.

https://conferences.iaea.org/indico/event/98/session/20/contribution/633/material/paper/0.pdf [25] W. Guo, L. Wang and G. Zhuang Nucl. Fusion 57056012 (2017) 